\title{
Rapid amplification of genetically modified organisms using a circular ferrofluid-driven PCR microchip
}

\author{
Yi Sun ${ }^{a}$, Yien-Chian Kwok ${ }^{a}$, Peter Foo-Peng Lee ${ }^{a}$, and Nam-Trung Nguyen ${ }^{b}$ \\ ${ }^{a}$ National Institute of Education, Nanyang Technological University, 1 Nanyang Walk, Singapore \\ 637616 \\ b School of Mechanical and Aerospace Engineering, Nanyang Technological University, 50 \\ Nanyang Avenue, Singapore, 639798 \\ * Corresponding author. Tel.: +65 67903836; Fax: +65 68969432. \\ E-mail address: yienchian.kwok@nie.edu.sg (Y.C. Kwok)
}

\begin{abstract}
The use of genetically modified organisms (GMOs) as food and in food products is becoming more and more widespread. Polymerase chain reaction (PCR) technology is extensively used for detection of GMOs in food products in order to verify compliance with labelling requirements. In this paper, we present a novel close-loop ferrofluid-driven PCR microchip for rapid amplification of GMOs. The microchip was fabricated in polymethyl methacrylate (PMMA) by $\mathrm{CO}_{2}$ laser ablation and was integrated with three temperature zones. PCR solution was contained in a circular closed microchannel and was driven by magnetic force generated by an external magnet through a small oil-based ferrofluid plug. Successful amplification of genetically modified soya and maize were achieved in less than 13 mins. This PCR microchip combines advantages of cycling flexibility and quick temperature transitions associated with two existing microchip PCR techniques, and it provides a cost saving and less time-consuming way to conduct preliminary screening of GMOs.
\end{abstract}

Keywords: Genetic modified organism (GMO); Ferrofluid; PCR microchip; PMMA 
Genetically modified organisms (GMOs) have been approved by several countries, such as US, European Union (EU), and Japan, etc [1]. The two most cultivated GMOs are maize and soya, which represent the staple constituents of many foods. In the past few years, different polymerase chain reaction (PCR)-based methods for the specific detection of the most economically important GMOs have been proposed [2]. A molecular screening method based on multiplex-PCR that involves amplification of specific soya or maize sequences from plant DNA and the amplification of 35S promoter and NOS terminator for the detection of genetically modified soya and maize was developed [3]. Conventionally, PCR is done in benchtop instruments, whose large thermal mass limits the speed at which thermal cycling can be carried out, resulting in reaction times on the order of a couple of hours or more. Therefore, considerable effort has been expended in miniaturizing this reaction.

Microchip PCR offers an ideal approach for decreasing the time PCR takes by providing devices with low thermal inertia, cycle times may be reduced to a few minutes [4]. Two basic types of PCR devices have been described, namely stationary PCR devices and continuous-flow (CF) PCR devices [5]. In a stationary PCR device, the sample solution is introduced into a small chamber. Thermal cycling is realized by heating and cooling the reaction chamber repeatedly according to required temperatures of the denaturing, annealing and extension processes. Such small chambers are difficult and expensive to fabricate, the temperature control system is usually bulky and the heating and cooling rates are relatively low [6]. For CF PCR devices, the PCR reaction mixture is pumped into a serpentine microfluidic channel and passes repeatedly through three spatially fixed temperature zones. Several CFPCR systems employing various pumping mechanisms were reported [7-12]. However, the use of pumps increases cost and imposes high requirement on microchip bonding integrity due to the application of high pressure. Other significant limitations of CFPCR devices include the large footprint of microchip and the fixed cycle number which is dictated by the channel layout. 
To facilitate widespread use of microfluidic PCR technology for GMO detection, a reliable and less expensive protocol for rapid amplification is urgently needed. In this paper, we demonstrated a novel close-loop magnetically-actuated microchip for rapid PCR. The PCR solution and a small oilbased plug of ferrofluid were contained in a circular closed microchannel integrated with three temperature zones. Magnetic force was generated by an external magnet through the ferrofluid plug situated besides the PCR solution. The neighbouring PCR solution was driven by the magnetic force and moved around the loop continuously. This microchip combines the cycling flexibility of the stationary chamber PCR and the quick temperature transitions associated with the continuous-flow PCR. Moreover, it has much simpler design and smaller footprint compared to most micro PCR devices, and using magnet as the driving force provides the advantages of low cost, small power consumption and low requirement on microchip bonding integrity.

Fig. 1 (a) shows a schematic diagram of the system set-up which includes three circulararranged copper heating blocks, an external permanent magnet and a ferrofluid driven PCR microchip. Each copper block is $55 \mathrm{~mm}$ high and $10 \mathrm{~mm}$ thick. Temperature can be set from $25{ }^{\circ} \mathrm{C}$ to $150{ }^{\circ} \mathrm{C}$. The copper blocks are separated by small air gaps to ensure the formation of three distinct and steady temperature zones. Unlike the parallel arrangement of the temperature zones in most flow-through microfluidic chips, the circular arrangement of the three zones allows the real realisation of the sequence of denaturation, annealing and extension instead of denaturation, extension and annealing. This prevents the denatured single-stranded DNA to re-hybridise in the extension zone, resulting in increased amplification efficiency. A cylindrical neodymium magnet (15 mm high and $10 \mathrm{~mm}$ in diameter) coupled with a home-made stepper motor was mounted on top of the circular microchannel. Both the rotation speed and number of rotation cycles of the magnet were programmable. 
The design of the microchip was much simpler compared to the rectangular serpentine CFPCR devices. It consists of a deep close-loop circular channel for PCR reaction and two shallow straight channels tangent to the circle for sample injection and collection. The PCR chip was fabricated in polymethyl methacrylate (PMMA) substrate by $\mathrm{CO}_{2}$ laser ablation [13]. To form the microfluidic device, the engraved substrate was then bonded together with a cover plate by a low pressure, high temperature thermal bonding technique under pressure of $20 \mathrm{kPa}$ at $165{ }^{\circ} \mathrm{C}$ for 30 minutes. As shown in Fig. 1 (b), the outer dimension of the chip was $30 \mathrm{~mm} \times 30 \mathrm{~mm}$. The circular channel was $62.8 \mathrm{~mm}$ long (with a diameter of $20 \mathrm{~mm}$ ), $900 \mu \mathrm{m}$ wide and $400 \mu \mathrm{m}$ deep, and each temperature zone was $21 \mathrm{~mm}$ long. Rectangular holes labelled as "thermal gaps”, were machined in a radial fashion to ensure proper heat isolation between different temperature zones. Access to the microchannel was provided by Teflon tubing attached to two holes drilled into the cover substrate.

The ferrofluid-driven PCR microchip was employed to amplify specific soya and maize sequences from plant DNA. Certified reference Roundup Ready soybeans and Bt-176 maize materials were purchased from Sigma (MO, USA). These were freeze-dried powders with 5\% GMO/non-GMO (w/w) soya and maize. The soybean and maize DNA were extracted from $100 \mathrm{mg}$ of each sample using Wizard genetic DNA purification kit (Promega, WI, USA). The 172-bp 35S-promoter sequence is specific for the detection of genetic modifications in soybeans. The 118-bp lectin gene $L E 1$ serves as a soybean control and is detectable in both transgenic and conventional soybeans. The 211-bp cryIA gene is for the specific identification of transgenic maize. The 226-bp invertase control gene serves as maize control. The details of primers were described elsewhere [14]. The $9 \mu \mathrm{L}$ reaction mixtures

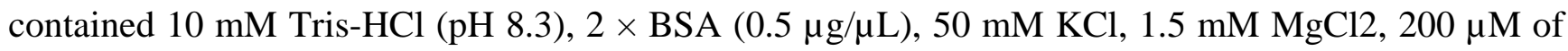


each dNTP, $1 \mu \mathrm{M}$ of each primer and $0.025 \mathrm{U} / \mu \mathrm{L}$ Taq DNA polymerase. $1 \mu \mathrm{L}$ different DNA were added separately as PCR template.

Before operation, the temperatures of the denaturation, annealing and extension zones were set at $95{ }^{\circ} \mathrm{C}, 62{ }^{\circ} \mathrm{C}$ and $72{ }^{\circ} \mathrm{C}$, respectively [14]. The microchip was placed on top of the heating blocks. The PCR mixture was injected using a glass syringe (Hamilton, NV, USA) through Teflon tube (Cole Parmer, Illinois, USA) fitted to the chip inlet hole. As shown in Fig. 2 (a), after the channel was completely filled, a small portion ( $2 \mu \mathrm{l})$ of ferrofluid (Ferrotec, CA, USA) was injected through the same inlet port. Ferrofluid is a stable colloidal suspension of sub-domain magnetic particles in a carrier fluid which can be moved through microchannels and adopt any geometry. The carrier liquid is synthetic ester oil, which is immiscible with the aqueous PCR reaction mixture. In a gradient field, the whole fluid responds as a homogeneous magnetic liquid which move to the region of the highest flux, so that ferrofluid can be precisely positioned and controlled by an external magnetic field [15].

Upon introduction of magnetic field, the ferrofluid plug immediately moved with the rotating magnet. PCR reaction mixture was pushed around the circular channel and flowed through the three temperature zones continuously. The four DNA fragments were amplified subsequently using the PCR microchip. The magnet speed was kept constant at $2.3 \mathrm{~mm} / \mathrm{s}$, and 25 cycles were completed within 13 mins. The time was greatly reduced compared to almost 2 hours when using conventional thermal cycler. Subsequent to each run, the microchip was washed by DI water and negative control of PCR was done to make sure that there was no carryover. The amplified DNA samples were analyzed using a 1.2\% crosslinked agarose gel (Sigma Chemical Co, MO, USA) stained with Ethidium Bromide (EtBr) (Sigma Chmeical Co, MO, USA). $0.5 \mu \mathrm{g}$ of 100 bp DNA ladder (500 $\mu \mathrm{g} / \mathrm{ml})$ was run together with PCR products. 
UV image of amplicons is shown in Fig. 2 (b). It can be seen that all the four genes were successfully amplified. The products with different length were clearly distinguished in the gel from each other and no amplification attributed to cross-contaminations were detected. As the 172 bp and 211 bp amplicons appeared only in 5\% transgenic soybean and maize samples, fluorescent intensities of these two bands were much less than those for the 118 bp and 226 bp amplicons that were detectable in both transgenic and conventional samples. The results show that PCR products obtained by the ferrofluid-driven PCR microchip faithfully reflected the initial number of genome copies. To improve sample throughput, a series of concentric circles can be designed on one microchip. As the dimension of microchannel is small compared to the magnet, one magnet is adequate to drive PCR reaction mixtures in all the microchannels. For more efficient and convenient product detection, direct coupling of the device to sensitive analytical systems is feasible, and online fluorescence detection by integrating a light source and a detector with the microchip is under investigation.

In conclusion, we demonstrated a close-loop magnetically-actuated microchip for rapid PCR amplification of GMO samples. Actuation of PCR mixtures was realized by a single button-sized external magnet through ferrofluid plugs inside the microchannels. Successful amplification of genetically modified soya and maize were achieved in less than 13 mins. This PCR microchip combines advantages of cycling flexibility and quick temperature transitions associated with two existing microchip PCR techniques, and it provides a cost saving and less time-consuming way to conduct preliminary screening of GMOs. 


\section{References}

1. Alary R, Serin A, Maury D, Jouira HB, Sirven JP, Gautier MF, Joudrier P (2002) Food Control 13: $235-244$.

2. Lin HY, Chiueh LC, Shih DYC (2000) Journal of Food and Drug Analysis 8: 200-207.

3. Forte VT, Pinto AD, Martino C, Tantillo GM, Grasso G, Schena FP (2005) Food Control 16: 535-539.

4. Zhang CS, Xu JL, Ma WL, Zheng WL (2006) Biotechnology Advances 24: 243-284.

5. Nguyen NT, Wereley ST (2006) Fundamentals and Applications of Microfluidics 457.

6. Lee DS, Park SH, Chung KH, Yoon TH, Kim SJ, Kim K, Kim YT (2004) Lab. Chip 4: 401407.

7. $\quad$ Obeid PJ, Christopoulos TK, Crabtree HJ, Backhouse CJ (2003) Anal. Chem. 75: 288-295.

8. Curcio M, Roeraade J (2003) Anal. Chem. 75: 1-7.

9. Belgrader P, Elkin CJ, Brown SB, Nasarabadi SN, Langlois RG, Milanovich FP (2003) Anal. Chem. 75: 3446-3450.

10. Chou CF, Changrani R, Roberts P, Sadler D, Burdon J, Zenhausern F (2002) Microelectro. Eng. 61: 921-925.

11. Chou HP, Unger MA, Quake SR (2001) Biomed. Microdev. 3: 323-330.

12. Sun Y, M.V.D S, Kwok YC, Nguyen NT (2008) Sens. Actuators B.

13. Sun Y, Kwok YC, Nguyen NT (2006) J. Micromech. Microeng. 16: 1681-1688.

14. Jankiewicz A, Broll H, Zagon J (1999) Eur. Food Res. Technol. 209: 77-82.

15. Sun Y, Kwok YC, Nguyen NT (2007) Lab Chip 7: 1012-1017. 
Fig. 1 (a) Schematic drawing of the PCR microchip with heating blocks and an external magnet. (b) Photograph of circular micro PCR device fabricated in PMMA by $\mathrm{CO}_{2}$ laser ablation.

Fig 2. (a) Concept of ferrofluid driven PCR chip. PCR reaction mixture, pushed by ferrofluid plug, flew through the three temperature zones continuously in the circular channel. (b) UV image of amplified soybean and maize samples. The magnet speed was kept constant at $2.3 \mathrm{~mm} / \mathrm{s}$, giving a cycle time of $27 \mathrm{~s}$. Triplicate amplifications were done with relative standard derivation less than $4 \%$. Lane 1: 100 bp DNA ladder. Lane 2: 172-bp 35S-promoter gene for genetic modified soybeans. Lane 3: 118-bp lectin gene $L E 1$, detectable in transgenic as well as in conventional soybeans. Lane 4: 211-bp cryIA gene for transgenic maize samples. Lane 5: 226-bp invertase control gene, detectable in transgenic as well as in conventional maize. 


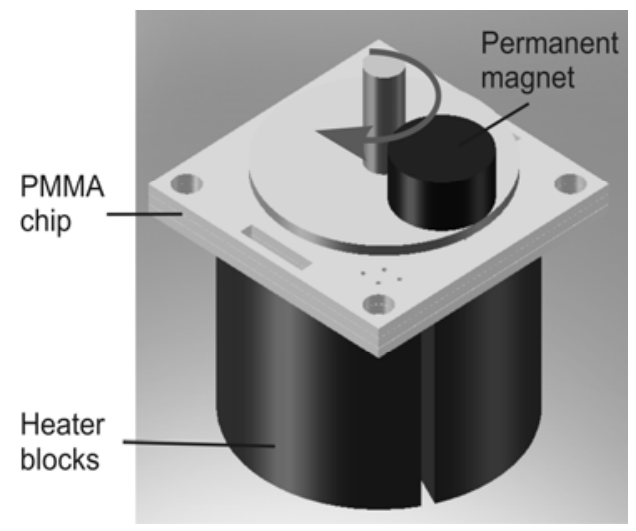

(a)

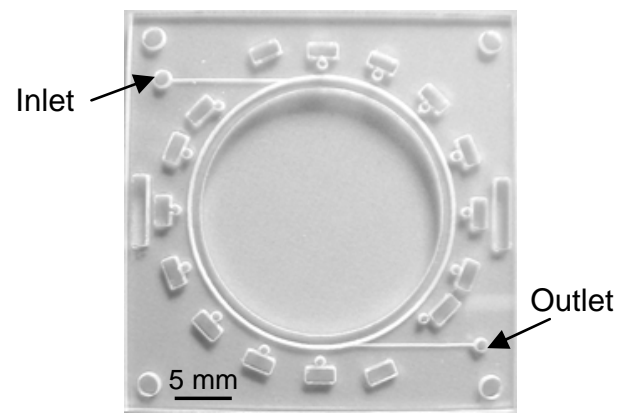

(b)

Fig. 1 (a) Schematic drawing of the PCR microchip with heating blocks and an external magnet. (b) Photograph of circular micro PCR device fabricated in PMMA by $\mathrm{CO}_{2}$ laser ablation. 


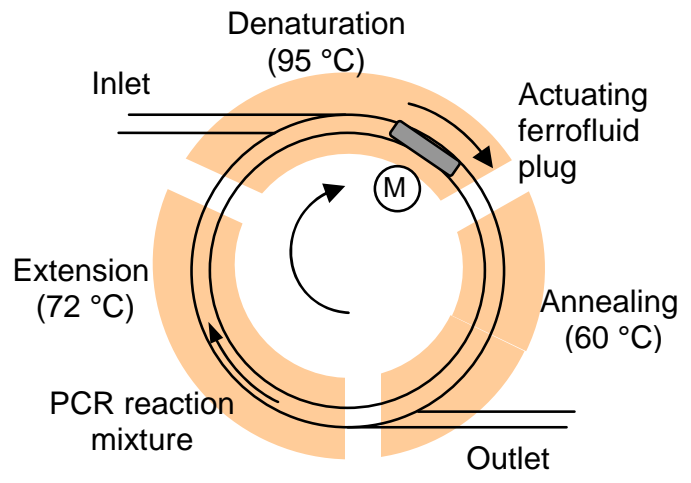

(a)
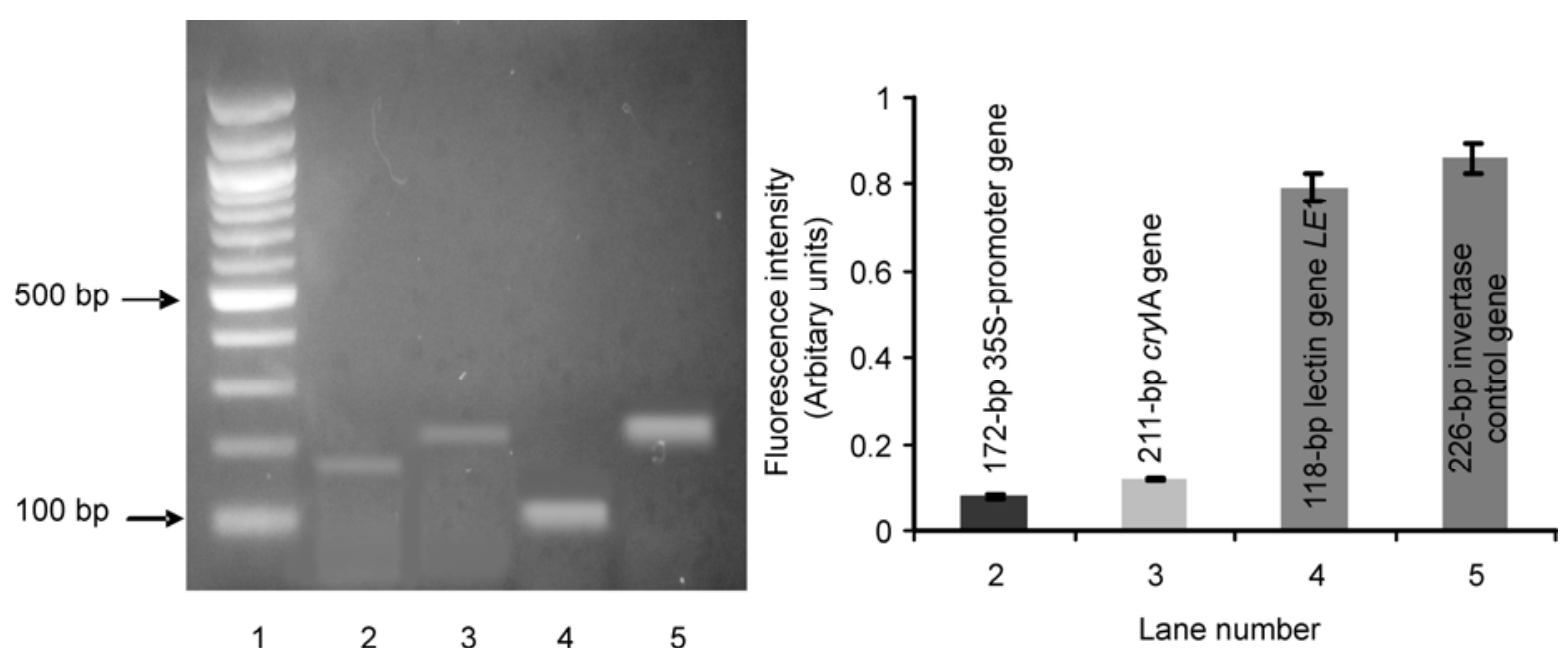

(b)

Fig 2. (a) Concept of ferrofluid driven PCR chip. PCR reaction mixture, pushed by ferrofluid plug, flew through the three temperature zones continuously in the circular channel. (b) UV image and relative fluorescence intensities of amplified soybean and maize samples. The magnet speed was kept constant at $2.3 \mathrm{~mm} / \mathrm{s}$, giving a cycle time of $27 \mathrm{~s}$. Triplicate amplifications were done with relative standard derivation less than 4\%. Lane 1: 100 bp DNA ladder. Lane 2: 172-bp 35S-promoter gene for genetic modified soybeans. Lane 3: 211-bp cryIA gene for transgenic maize samples. Lane 4: 118-bp lectin gene $L E 1$, detectable in transgenic as well as in conventional soybeans. Lane 5: 226-bp invertase control gene, detectable in transgenic as well as in conventional maize. Band intensities were normalized with respect to the $500 \mathrm{bp}$ fragment of the 100 bp ladder. 